\title{
Malaria transmission modelling: a network perspective
}

\author{
Jiming Liư ${ }^{*}$, Bo Yang ${ }^{2}$, William K Cheung ${ }^{1}$ and Guojing Yang ${ }^{3}$
}

\begin{abstract}
Malaria transmission can be affected by multiple or even hidden factors, making it difficult to timely and accurately predict the impact of elimination and eradication programs that have been undertaken and the potential resurgence and spread that may continue to emerge. One approach at the moment is to develop and deploy surveillance systems in an attempt to identify them as timely as possible and thus to enable policy makers to modify and implement strategies for further preventing the transmission. Most of the surveillance data will be of temporal and spatial nature. From an interdisciplinary point of view, it would be interesting to ask the following important as well as challenging question: Based on the available surveillance data in temporal and spatial forms, how can we build a more effective surveillance mechanism for monitoring and early detecting the relative prevalence and transmission patterns of malaria? What we can note from the existing clustering-based surveillance software systems is that they do not infer the underlying transmission networks of malaria. However, such networks can be quite informative and insightful as they characterize how malaria transmits from one place to another. They can also in turn allow public health policy makers and researchers to uncover the hidden and interacting factors such as environment, genetics and ecology and to discover/predict malaria transmission patterns/trends. The network perspective further extends the present approaches to modelling malaria transmission based on a set of chosen factors. In this article, we survey the related work on transmission network inference, discuss how such an approach can be utilized in developing an effective computational means for inferring malaria transmission networks based on partial surveillance data, and what methodological steps and issues may be involved in its formulation and validation.
\end{abstract}

\section{Multilingual abstracts}

Please see Additional file 1 for translations of the abstract into the six official working languages of the United Nations.

\section{Background}

Malaria transmission is challenging to model; its vector can be quite complex due to topographical and climatic variations as well as human mobility [1]. One of the United Nations (UN) Millennium Development Goals is to "have halted by 2015 and begun to reverse the incidence of malaria" which annually causes $\sim 1$ million death or $>1$ death every 30-60 second [2,3]. World Health Organization (WHO) has suggested that the most important measure is a timely response with the implementation of effective interventions once it has been

\footnotetext{
* Correspondence: jiming@comp.hkbu.edu.hk

${ }^{1}$ Department of Computer Science, Hong Kong Baptist University, Kowloon

Tong, Hong Kong

Full list of author information is available at the end of the article
}

detected [4]. This requires an effective monitoring/surveillance system that can provide long range forecasting, early warning, and early detection [5]. Towards this end, malaria transmission patterns will be informative in performing such surveillance functions.

This article provides a review of related work on how to develop a computational means for inferring malaria transmission networks in populations, which incorporates: (1) partial surveillance data over time, i.e., the temporal-spatial distributions of cases of infection, and (2) infection models of malaria. A transmission network to characterize the temporal-spatial patterns of disease transmission, or a temporal-spatial disease transmission network, consists of a set of nodes and a set of links that connect them, where the nodes correspond to spatial locations, such as villages, with reported/observed disease incidences over time, and the directional links connecting the nodes correspond to the probability/ likelihood of disease "transmission" from one node to

\section{Biomed Central}


another over time, e.g., hidden pathways of malaria transmission.

Technically, the problem of computationally inferring malaria transmission networks is both interesting and challenging because, during the process of disease spread, the reported infection cases do not directly reflect the full extent of transmission, nor the underlying transmission patterns. It would be desirable for us to detect such networks from the partially available surveillance data. In doing so, we may incorporate a malaria infection model, e.g., the Ross-MacDonald model [6].

In this article, we discuss how such a computational method differs from existing methods of network inference, in the light of the unique nature of malaria transmission dynamics. In computer science, related studies have been carried out to solve the problem of inferring information diffusion networks from Web data [7-10]. These studies only consider temporal information and cannot readily be extended sufficiently to incorporate additional information, such as spatial, environmental, climatic, and clinical information. Also, most of the methods are based on independent cascading models, assuming that one node will be independently infected by others with respective probabilities, and cannot readily integrate more complicated infection/propagation models.

\section{Methods}

We searched and reviewed the related research papers in (1) bibliographical databases including Web of Science and PubMed, (2) international conferences including ACM-SIGKDD, ICDM, ICML, SIAM-SDM, WWW, etc., and (3) World Health Organization (WHO) reports. The aim of our survey is to find and study (1) existing methods for modelling disease infections and epidemicslike transmission processes based on structural representations such as transmission paths or networks, and furthermore (2) those for inferring the underlying transmission networks based on temporal and/or spatial surveillance data.

\section{Scan statistics-based clustering and network-based epidemic dynamics modelling}

We first examined existing studies on modelling temporal-spatial patterns of epidemic dynamics. We started by evaluating the scan statistics-based clustering methods and their related software tools for modelling malaria transmission (which are also useful for detecting active foci or hotspots over time and space). Our survey aims to identify the need for a more explicitly structured representation of disease spread, e.g., the interrelationships among different locations due to the heterogeneous temporal-spatial factors affecting hosts, vectors, and parasites at various scales. Such a representation would be particularly desired in planning cost-effective intervention strategies. We then surveyed the related studies in both epidemiology and other disciplines that had demonstrated how disease spread and/or information dynamics could be revealed based on network representations, i.e., disease spread dynamics on networks. In doing so, we focused on how dynamics may vary with respect to the characteristics of networks, e.g., regular, small-world, or scale-free networks, as well as human behaviour, e.g., mobility.

\section{Inferring networks from temporal data}

Once we confirmed the role of networks in understanding disease spread dynamics, our next logical step was to investigate how existing studies had attempted to predict the structures of underlying transmission networks, whether indirectly, e.g., through information on human mobility and social contact activities, or directly, e.g., based on observed/reported cases of infection. We paid special attention to the present methods that had been implemented for the purpose of inferring an underlying transmission network (links) from its observed (node) activities. The surveyed work, although may have appeared in the fields other than epidemiology, would provide us with a good understanding of the general methodology for detecting interaction networks from observations over time and space.

\section{Findings and discussion}

The key concepts, their corresponding representative examples, and publications that we reviewed are summarized in Table 1. As depicted in Figure 1, they constitute four distinct approaches. In what follows, we will present our findings and observations.

\section{Scan statistics-based clustering and network-based epidemic dynamics modeling}

Malaria transmission can be affected by multiple factors, such as biology, environment, and socio-economy, that directly impinge on the interactions among hosts, vectors, and parasites at varying degrees and scales [17-19]. A feasible means to model malaria transmission is to rely on passive case reporting surveillance systems or surveys through local, national, or regional public health and medical organizations. Most of the data collected from such systems or surveys may contain temporal, spatial, clinical, and/or demographic information, and may cover only arbitrary locations and age-groups. At the moment, temporal-spatial scan statistics-based clustering techniques have been applied to the analysis and characterization of temporal-spatial patterns of malaria [11]. In doing so, software tools [12-15] have been used to manage and geographically map reported malaria cases, and to test whether the cases are randomly or 
Table 1 A summary of key concepts, representative examples, and corresponding references

\begin{tabular}{|c|c|c|}
\hline Key concepts and considerations & Representative examples & References \\
\hline \multirow[t]{3}{*}{ 1. Temporal-spatial characterization } & Scan statistics-based clustering & [11] \\
\hline & Scan software tools & [12-15] \\
\hline & Other applications (active foci or hotspots) & [16] \\
\hline \multirow[t]{3}{*}{ Related factors } & $\begin{array}{l}\text { Biology, environment, and socio-economy } \\
\text { affecting interactions among hosts, vectors, } \\
\text { and parasites at various scales }\end{array}$ & [17-19] \\
\hline & $\begin{array}{l}\text { Entomological inoculation rates, vector capacity, } \\
\text { or force of infection }\end{array}$ & {$[20]$} \\
\hline & $\begin{array}{l}\text { A combination of epidemiological, geographical, } \\
\text { and demographic factors }\end{array}$ & [21] \\
\hline \multirow[t]{5}{*}{$\begin{array}{l}\text { 2. Modelling disease and/or information } \\
\text { dynamics on networks }\end{array}$} & $\begin{array}{l}\text { Dynamics of infectious diseases on regular, } \\
\text { small-world, or scale-free networks }\end{array}$ & {$[22-27]$} \\
\hline & $\begin{array}{l}\text { Critical value analysis of typical epidemics on } \\
\text { complex network }\end{array}$ & [28-33] \\
\hline & $\begin{array}{l}\text { Diffusion of rumours or innovation on } \\
\text { social networks }\end{array}$ & [34-36] \\
\hline & $\begin{array}{l}\text { Viral marketing and recommendation } \\
\text { strategies }\end{array}$ & {$[37-39]$} \\
\hline & $\begin{array}{l}\text { Cascading in virtual blog spaces, and their } \\
\text { propagation trends }\end{array}$ & {$[10,40-43]$} \\
\hline \multirow[t]{2}{*}{ Related factors } & Alternative spatial representations & [44] \\
\hline & $\begin{array}{l}\text { Effects of human mobility on the dynamics } \\
\text { of disease transmission }\end{array}$ & [45] \\
\hline \multirow{3}{*}{$\begin{array}{l}\text { 3. Understanding the structures of underlying } \\
\text { transmission networks via indirect means }\end{array}$} & Population travelling and mobility patterns & {$[46,47]$} \\
\hline & Social contact activities & {$[48-50]$} \\
\hline & Sexual relationships & [51] \\
\hline \multirow[t]{2}{*}{ 4. Inferring transmission parameters from data } & $\begin{array}{l}\text { EM-based estimation algorithm to infer daily } \\
\text { transmission rate between households }\end{array}$ & [52] \\
\hline & $\begin{array}{l}\text { Markov Chain Monte Carlo (MCMC) method to } \\
\text { estimate transmission parameters }\end{array}$ & [53] \\
\hline \multirow[t]{7}{*}{ 5. Inferring an underlying network from data } & $\begin{array}{l}\text { Social networks based on the interpersonal } \\
\text { interaction records }\end{array}$ & {$[54-58]$} \\
\hline & Interaction networks between proteins in a cell & {$[59,60]$} \\
\hline & Supervised classification & [7] \\
\hline & Expectation-maximization (EM)-like algorithm & [10] \\
\hline & Narrow and deep tree-like structure analysis & [8] \\
\hline & Likelihood-maximization & [9] \\
\hline & Independent cascading models & [41] \\
\hline \multirow[t]{5}{*}{ 6. Computational issues } & Conventional optimization methods & [61] \\
\hline & $\begin{array}{l}\text { Potentially large-scale and/or dynamically-evolving } \\
\text { surveillance data, e.g., over decades of temporal intervals }\end{array}$ & {$[62-64]$} \\
\hline & Different levels of spatial categories & {$[62,63]$} \\
\hline & Multiple environmental or biological factors incorporated & {$[19,64]$} \\
\hline & Alternative AOC methods & {$[65-67]$} \\
\hline
\end{tabular}

significantly distributed with spatial or space-time disease clusters. Sometimes, for a more accurate malaria map, additional information may be incorporated, e.g., by means of modelling entomological inoculation rates, vector capacity, or force of infection [20], or by using a combination of epidemiological, geographical, and demographic data [21]. Generally speaking, such temporal-spatial techniques do not infer the underlying transmission patterns (modelled as networks) of malaria. Such transmission networks can be informative and 


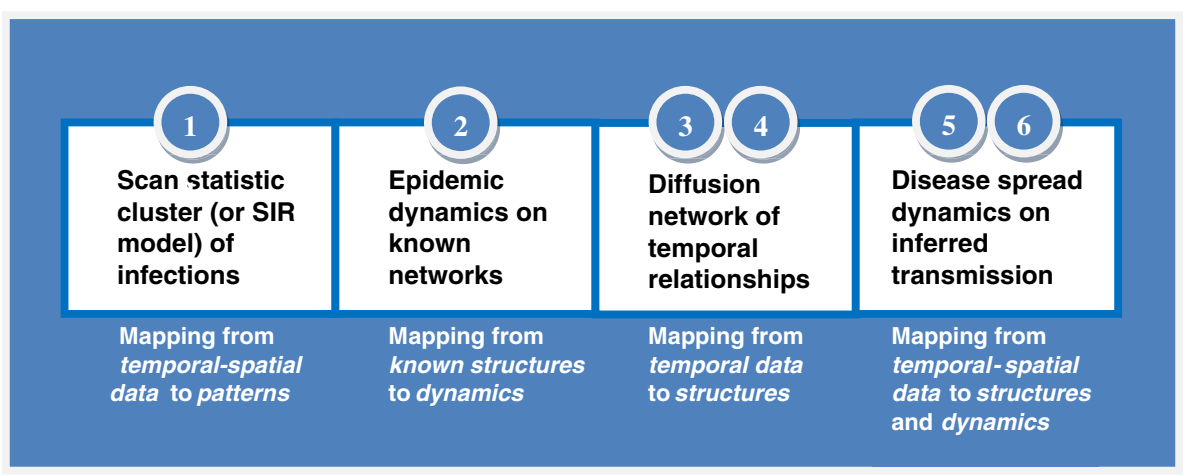

Figure 1 The four approaches (as shown in the four boxes) discussed in this article are listed by highlighting their distinct characteristics. The labelled numbers correspond to the section numbers in Table 1.

perceptive, as they characterize how malaria is diffused or transmitted from one location to another (e.g., across villages) over time, providing a new way of detecting the active foci or hotspots of malaria transmission other than directly estimating epidemiological factors or identifying clusters with above-average transmission intensity [16]. Therefore, by integrating both temporal-spatial clusters of cases of infection and temporal-spatial transmission networks of malaria, existing local, national, or regional surveillance systems can further be enhanced in their functional capacities of predicting and analyzing the impact of malaria transmission and their underlying factors, as well as evaluating existing intervention or eradication strategies and guiding new control efforts.

Next, we survey related studies on modelling the dynamics of epidemics on networks. Although the subjects involved in different epidemics can vary considerably, many can be modelled by either SIR/SIS models [68-70], cascading models [37], or threshold models [71,72]. The assumption behind the basic SIR or SIS models is that an individual in a population will be in one of the three states: suspected (S), infected (I), and recovered (R). If individuals are viewed as nodes, and the contacts between them as links, a network can be obtained that describes who will infect whom with what probabilities based on the SIR or SIS model. Grassberger first studied the dynamics of infectious diseases that propagate on regular networks using the percolation theory [22]. Studies have revealed that many real-world networks, including social networks in which infectious diseases propagate [26,27], are either small-world [23] or scalefree $[24,25]$, rather than regular or random, as thought previously [73].

As the underlying structures of networks will influence the effect that dynamics of epidemics will have on them, researchers, such as Pastor-Satorras and Vespignani, have made several contributions to critical value analysis of typical epidemics on different types of complex network [28-33]. Based on the mean-field theory, they found that, compared with homogeneous networks, scale-free networks are fragile to the invasion of infectious diseases, computer viruses, or any other type of epidemics. In addition, researchers have also considered different spatial representations in modelling directly transmitted infectious diseases [44] and the effects of human mobility on the dynamics of disease transmission on networks [45].

Epidemics on networks have also been studied in various disciplines. Sociologists are concerned with the diffusion of rumours or innovation on social networks [34-36]; economists have studied viral marketing and recommendation strategies by considering cascading dynamics as well as the network effects of vital nodes [37-39]; computer scientists are interested in how some topics can quickly cascade in virtual blog spaces, and their propagation trends $[10,40,42,43]$.

As can be noted, existing studies have contributed to the modelling of disease and/or information dynamics on networks. However, most of them have made a strong assumption that the structures of underlying networks, over which a disease spreads, are known beforehand. This may not hold in the real world, as the structures allowing the underlying transmission networks to emerge will not be known directly (even though in some cases, indirect means have been used, e.g., based on population travelling and mobility patterns [46,47], social contact activities [48-50], or sexual relationships [51]); what may be obtained is only the time when particular nodes are found infected, but not how they get infected, nor how they affect their neighbouring areas. Then, the question that remains unanswered is how to model disease spread dynamics on hidden networks, which in turn leads to the basic question of how to computationally infer such hidden transmission networks based on partial temporal-spatial surveillance data.

\section{Inferring networks from temporal data}

Inferring underlying transmission and propagation processes by analyzing the observable spreading patterns has 
drawn special attention from several domains, ranging from the path inference of information propagation $[7,9,54]$, the construction of social networks based on the interpersonal interaction records [55-58], to the inference of interaction networks between proteins in a cell $[59,60]$. Adar and Adamic [7] first studied the problem of inferring information dynamics in blog space, and formulated the diffusion-network prediction problem as a supervised classification problem. Gruhl et al. [10] developed a method for inferring how topics spread among the blogs and proposed an expectation-maximization (EM)-like algorithm for estimating propagation probabilities over an underlying diffusion network. Liben-Nowell and Kleinberg [8] studied information flow on a global scale by analyzing chain-letter datasets, and indicated that the propagations of chain letters spread on social networks are shaped by narrow and deep tree-like structures with most one-child nodes. Gomez-Rodriguez et al. [9,74] formulated the problem of inferring a diffusion network into a likelihoodmaximization task, and proposed a greedy algorithm that can approximately learn underlying networks from observed infection sequences.

In addition to the above, a few studies have been carried out in the domain of epidemiology. Zelner et al. [52] examined how infections spread after the pointsource outbreaks of secondary norovirus transmission between households by analyzing a real-world dataset that described a food-borne norovirus outbreak in 30 daycare centres in Stockholm. They extended the basic SIR model by explicitly considering the incubation period of norovirus, and correspondingly proposed an EM-based estimation algorithm to infer daily transmission rate, and the means and shapes of the Poisson distributions of incubation and infectious periods. Similarly, Hohle et al. [53] proposed an inference technique based on the Markov Chain Monte Carlo (MCMC) method to estimate transmission parameters for an infectious disease from time series data by allowing variability in the incubation period.

To date, most of the existing work has mainly focused on how to use temporal information to infer underlying transmission networks, but neglected spatial information, which would be equally critical in shaping the underlying networks. To the best of our knowledge, almost none of them have explicitly studied the problem of designing a surveillance mechanism based on the idea of inferring disease transmission networks and corresponding dynamics from collected data through the integration of both temporal and spatial information.

\section{Towards network-based malaria transmission modelling}

Further to the above review of the related work, we next discuss a potential methodology for developing a computational means of inferring disease transmission networks based on partial temporal-spatial surveillance data.

\section{Inferring transmission networks from temporal-spatial surveillance data}

First of all, it should be pointed out that although the idea of inferring the underlying networks of disease transmission from surveillance data can benefit from the related methods in computer science [7-10,74], the former differs from the latter in some fundamental ways, in the light of the unique nature of disease transmission dynamics:

(1) The existing methods mainly focus on learning information diffusion networks from Web data, and thus when inferring the structures of diffusion networks, most of them merely consider temporal information, and neglect spatial, environmental, climatic, demographic, clinical, and other key factors as related to malaria transmission.

(2) Most of them are based on independent cascading models [7-9,25,41,43,74] that are widely used to characterize information flows, with the assumption that one node will be independently infected by others with respective probabilities. However, in the real world, infectious diseases follow much more complicated infection/propagation models such as the ones of malaria transmission [75]. Reasonable transmission networks may be discovered only when their corresponding infection/propagation models are considered sufficiently during the course of formulation and computation.

In view of the above, it is clear that there exists a need for developing novel methods for modelling and inferring disease transmission networks from surveillance data that would enable us to incorporate temporal, spatial, as well as other recorded attributes. To do so, we need to design corresponding algorithms. An illustrative example of the probabilistic approach to modelling and inferring malaria transmission networks is given as follows.

Suppose that surveillance data on malaria consists of collected case observations that are formatted as $M$ temporal-spatial series (corresponding to $M$ locations, such as villages). Items in such a temporal-spatial series take the form of 4-tuple $\left(i, t_{i}, k_{i}\right.$, attr $)$, which indicates that $k_{i}$ cases at node $i$ with an attribute $a^{a t r_{i}}$ are reported/observed during a time span $t_{i}$. Note that node $i$ corresponds to a specific location, such as a village, whose geographic, environmental, climatic, demographic, or clinical attributes can be incorporated in $\operatorname{attr}_{i} . t_{i}$ may be measured in different scales, such as 
daily, weekly, or monthly. A probabilistic approach to network-based disease transmission modelling can readily be carried out based on the following steps:

Step 1. Let $\lambda$ be the infectious strength, which indicates how likely one node becomes infected by its neighbours, or in other words, how likely a disease propagates to one node from its neighbours. Drawing on the basic RossMacDonald model, the infectious strength of malaria at node $i$ during an interval $t_{i}$ (i.e., a disease infection/propagation model integrating geographic, environmental, climatic, demographic, and clinical information) is defined as:

$$
\lambda\left(B_{i}, t_{i}, k_{i}, \text { attr }_{i}\right)=\left(a^{2} b c m t_{i} / \mu\right) \times \sum_{j \in B_{i}} k_{j}
$$

where $B_{i}$ denotes the neighbours of node $i$ in network $N .1 / \mu$ denotes the life expectancy of mosquitoes, and $m$ corresponds to the number of mosquitoes per human host in location $i . a$, $b$, and $c$ indicate the rate of biting humans by a single mosquito, the proportion of infected bites in humans that produce an infection, and the transmission efficiency from humans to mosquitoes, respectively, which are empirically/ clinically determined for the endemic areas and populations in focus. $\sum_{j \in B_{i}} k_{j}$ denotes the total number of the infected cases within the neighbouring locations of node $i$.

Step 2. Compute the likelihood of the time series $r$ being observed as follows:

$$
\begin{aligned}
L_{r}(N \mid \lambda) & =\prod_{i=1}^{l_{r}} P\left(t_{i}<X_{i} \leq t_{i}+\Delta t\right) \\
& =\prod_{i=1}^{l_{r}} \lambda\left(B_{i}, t_{i}, k_{i}, a t t r_{i}\right) e^{-\lambda\left(B_{i}, t_{i}, k_{i}, a t r_{i}\right) t_{i}}
\end{aligned}
$$

where $N$ denotes an underlying transmission network, on which the disease spreads according to a disease-specific model $\lambda$, and $l_{r}$ is the length of the temporal-spatial series $r$.

Step 3. Given $M$ temporal-spatial series observed and an infection/propagation model $\lambda$, define and maximize an objective function based on the likelihood function and a penalty term to avoid over-fitting that happens when the model's degree of freedom is too higher with respect to the number of observed data:

$$
O(N)=L(N \mid \lambda)+\|N\|_{L_{1}}
$$

where $L(N \mid \lambda)=\prod_{r=1}^{M} L_{r}(N \mid \lambda)$ and $\|N\|_{L_{1}}$ is the $L_{1}$-norm term of the adjacency matrix of network $N$.
The optimization can be carried out by, for example, formulating $O(N)$ such that it is a submodular function with the property of diminishing return [63], and then using the submodular greedy method to determine an approximately optimal transmission network. To handle the case of network inference that potentially involves largescale and/or dynamically-evolving surveillance data, e.g., over decades of temporal intervals $[19,64,65]$, thousands of locations in different levels of spatial categories [64,65], and multiple environmental or biological factors considered $[65,66]$, alternative optimization methods can be considered; one of the possible ways is to utilize autonomyoriented computing (AOC) $[15,27,67]$. One can take further advantage of the autonomy aspect emphasized in the AOC paradigm and consider that some active surveillance strategies can be employed. Then, the optimization space will not span only the model parameter space but also the allowable surveillance strategy space so as to further boost to the accuracy of the transmission network inference.

To summarize, adopting such a network approach can be quite useful for public health authorities and epidemiologists to gain insights into the impacts of disease spread over time and space and the underlying factors (e.g., corresponding environmental/climatic factors, mosquito ecology/ genetic evolution, human mobility, and control/intervention strategies) in a region, e.g., through constructing and comparing a series of transmission networks.

\section{Empirical evaluation and application}

Finally, we comment on how to validate the method of inferring malaria transmission networks, particularly by testing it based on the available malaria dataset historically collected from various endemic areas.

We may validate the network-based modelling method by means of a cross-validation strategy. In doing so, we divide the dataset into two parts. The first part is used to determine the transmission network covering the area in focus. Based on the inferred transmission network and a malaria infection model, we simulate malaria transmission in the respective region based on the following steps: (1) initialize the onset of malaria propagation on the detected transmission network by selecting the nodes corresponding to the earlier reported cases in the real data; (2) in each iteration (each iteration corresponds to a time scale, e.g., weekly), each infected node tries to infect its neighbours (determined by the transmission network); (3) record the obtained temporalspatial distribution (times are denoted by the sequence of iterations and locations are denoted by the geographical attributes of nodes) of infected cases during the whole simulation for the covered period. Then, we compare the cases sampled by the aforementioned simulation with the real cases provided by the second part of 


\begin{tabular}{|c|c|}
\hline \multirow[t]{3}{*}{ Inference of transmission networks } & - Incorporating partial surveillance data over time, i.e., the temporal-spatial distributions of cases of infection \\
\hline & $\begin{array}{l}\text { - Constructing specific infection models of malaria, while incorporating additional information, } \\
\text { such as geographic, environmental, climatic, demographic, clinical, and behavioural information }\end{array}$ \\
\hline & $\begin{array}{l}\text { - Developing computational tractable probabilistic methods, as well as } \\
\text { extending the existing models proposed in computer science (e.g., independent cascading models) }\end{array}$ \\
\hline \multirow[t]{3}{*}{ Use of transmission networks } & - Validating inferred transmission networks by testing them with available malaria data \\
\hline & $\begin{array}{l}\text { - Predicting and analyzing the impact of malaria transmission and their underlying factors } \\
\text { over time and space through constructing and comparing a series of transmission networks }\end{array}$ \\
\hline & - Evaluating existing intervention or eradication strategies and guiding new control efforts \\
\hline
\end{tabular}

the dataset, and evaluate the utility of the inferred network in terms of some defined metrics. The divisions of the dataset could be performed using different ratios of learning data and testing data.

\section{Conclusion}

In view of the need for healthcare providers, government disease control and prevention organizations, and international agencies to predict and analyze the impacts of malaria transmission and their underlying factors, in this article we have discussed various approaches related to epidemic dynamics modelling and transmission mechanism/network inference from surveillance data, and have pointed out the needs for future research (see future research priorities in Table 2). In particular, we have addressed how to extend the existing computational concepts and methods of transmission networks to those of inferring and predicting the prevalence and transmission patterns of malaria in populations, and have outlined a potential methodology that would enable us to develop and apply a computational method to automatically infer the underlying malaria transmission network that utilizes (1) partial surveillance data, i.e., the temporal-spatial distributions of cases of infection, and (2) infection/propagation models of the disease.

\section{Additional file}

Additional file 1: Multilingual abstracts in the six official working languages of the United Nations.

\section{Competing interests}

The authors declare that they have no competing interests.

\section{Authors' contributions}

$J L$ and BY designed research; $J, B Y, W K C$ and GY performed research; $J L$ and BY wrote the paper; All authors read and approved the final manuscript.

\section{Acknowledgements}

The authors would like to acknowledge the funding support of Hong Kong Research Grants Council (RGC) for the work being presented in this article (HKBU211212). Bo Yang would also like to acknowledge the support of Chinese Program for New Century Excellent Talents in University (NCET-110204).

\section{Author details}

${ }^{1}$ Department of Computer Science, Hong Kong Baptist University, Kowloon Tong, Hong Kong. ${ }^{2}$ College of Computer Science and Technology, Jilin University, Changchun, China. ${ }^{3}$ The Jockey Club School of Public Health and Primary Care, Chinese University of Hong Kong, Hong Kong.

Received: 11 August 2012 Accepted: 11 October 2012

Published: 1 November 2012

\section{References}

1. Sutherst RW: Global change and human vulnerability to vector-borne diseases. Clin Microbiol Rev 2004, 17(1):136-173.

2. World Health Organization: Malaria. http://www.who.int/malaria/en.

3. United Nations: Millennium Development Goals (MDGs): Goal 6: Combat HIVI AIDS, Malaria and Other Diseases. http://www.who.int/topics/ millennium_development_goals/diseases/en/index.html.

4. World Health Organization: Malaria Control Today - Current WHO Recommendations. Geneva: WHO Working Document; 2005.

5. Guintran J, Delacollette C, Trigg P: Systems for the Early Detection of Malaria Epidemics in Africa - An Analysis of Current Practices and Future Priorities. Organization: World Health; 2006.

6. Ruan S, Xiao D, Beierc JC: On the delayed Ross-MacDonald model for malaria transmission. Bull Math Biophy 2008, 70(4):1098-1114.

7. Adar $E$, Adamic LA: Tracking information epidemics in blogspace. Proc IEEE/WIC/ACM-WI'05 2005, 207-214.

8. Liben-Nowell D, Kleinberg J: Tracing the flow of information on a global scale using Internet chain-letter data. Proc Nat Acad Sci 2008, 105(12):4633-4638

9. Gomez Rodriguez M, Leskovec J, Krause A: Inferring networks of diffusion and influence. Proc ACM-SIGKDD'10 2010, 5(4):1019-1028.

10. Gruhl D, Guha R, Liben-Nowell D, Tomkins A: Information diffusion through blogspace. Proc WWW'04 2004, :491-501.

11. Coleman M, Mabuza AM, Kok G, Coetzee M, Durrheim DN: Using the SaTScan method to detect local malaria clusters for guiding malaria control programmes. Malar J 2009, 8:68.

12. Kulldorff M: SaTScanTM User Guide for version8.0. 2009. http://www.satscan. org.

13. Robertson C, Nelson TA: Review of software for space-time disease surveillance. Int J Health Geogr 2010, 9:16.

14. Eisen L, Coleman M, Lozano-Fuentes S, McEachen N, Orlans M, Coleman M: Multi-disease data management system platform for vector-borne diseases. PLoS Negl Trop Dis 2011, 5(3):e1016.

15. Martinez-Piedra R, Loyola-Elizondo E, Vidaurre-Arenas M, Aguilar PN: Software programs for mapping and spatial analysis in epidemiology and public health. Epidemiol Bull 2004, 25(4):1-9.

16. Bousema T, Griffin JT, Sauerwein RW, Smith DL, Churcher TS, Takken W, Ghani A, Drakeley C, Gosling R: Hitting hotspots: spatial targeting of malaria for control and elimination. PLoS Med 2012, 9(1):e1001165.

17. malERA Consultative Group on Modeling: A research agenda for malaria eradication: modelling. PLoS Med 2011, 8(1):e1000403.

18. Bomblies A, Duchemin JB, Eltahir EA: A mechanistic approach for accurate simulation of village scale malaria transmission. Malar J 2009, 8:223.

19. Huang F, Zhou S, Zhang S, Zhang H, Li W: Meteorological factors-based spatio-temporal mapping and predicting malaria in central China. Am J Trop Med Hyg 2011, 85(3):560-567. 
20. Gemperli A, Vounatsou P, Sogoba N, Smith T: Malaria mapping using transmission models: application to survey data from Mali. Am J Epidemiol 2006, 163(3):289-97.

21. Snow RW, Guerra CA, Noor AM, Myint HY, Hay SI: The global distribution of clinical episodes of Plasmodium falciparum malaria. Nature 2005, 434(7030):214-7.

22. Grassberger P: On the critical behaviour of the general epidemic process and dynamical percolation. Math Biosci 1983, 63:157-172.

23. Watts DJ, Strogatz SH: Collective dynamics of small-world networks. Nature 1998, 393:440-442.

24. Barabasi AL, Albert R: Emergence of scaling in random networks. Science 1999, 286:509-512.

25. Wu F, Huberman BA, Adamic LA, Tyler JR: Information flow in social groups. Phys A 2004, 337:327-335.

26. Dangerfield CE, Ross JV, Keeling MJ: Integrating stochasticity and network structure into an epidemic model. J Roy Soc Interface 2009, 6(38):761-774.

27. Keeling MJ, Eames KTD: Networks and epidemic models. J Roy Soc Interface 2005, 2:295-307.

28. Pastor-Satorras R, Vespignani A: Epidemic dynamics and endemic states in complex networks. Phys Rev E 2001, 63:066117.

29. Pastor-Satorras $R$, Vespignani A: Epidemics and immunization in scale-free networks. Handbook of Graph and Networks: Wiley-VCH; 2003.

30. Kuperman M, Abramson G: Small world effect in an epidemiological model. Phys Rev E 2000, 62:7059-7064.

31. Newman MEJ, Jensen I, Ziff RM: Percolation and epidemics in a two dimensional small world. Phys Rev E 2002, 65:021904.

32. Pastor-Satorras $R$, Vespignani A: Epidemic dynamics in finite size scale-free networks. Phys Rev E 2002, 65:035108.

33. Boguna M, Pastor-Satorras $R$, Vespignani $A$ : Absence of epidemic threshold in scale-free networks with connectivity correlations. Phys Rev Lett 2003, 90:028701.

34. Rogers E: Diffusion of Innovations. New York: Free Press; 1995.

35. Yang J, Leskovec J: Modelling information diffusion in implicit networks. Proc ICDM'10 2010, 599-608.

36. Rogers EM: New product adoption and diffusion. J Consumer Res 1976 2:290-301

37. Goldenberg J, Libai B, Muller E: Talk of the network: a complex systems look at the underlying process of word-of-moth. Market Lett 2001, 12(3):211-223.

38. Leskovec J, Adamic L, Huberman B: The dynamics of viral marketing. ACM Trans Web 2007, 1(1):1-39.

39. Leskovec J, Backstrom L, Kleinberg J: Meme-tracking and the dynamics of the news cycle. Proc ACM-SIGKDD'09 2009, 497-506.

40. Kumar R, Novak J, Raghavan P, Tomkins A: On the bursty evolution of blogspace. World Wide Web 2005, 8(2):159-178.

41. Leskovec J, McGlohon M, Faloutsos C, Glance NS, Hurst M: Patterns of cascading behaviour in large blog graphs. Proc SIAM-SDM'07 2007, 551-556.

42. Stewart $A$, Chen L, Paiu R, Nejdl W: Discovering information diffusion paths from blogosphere for online advertising. Proc ADKDD'07 2007, 46-54.

43. Sadikov E, Medina M, Leskovec J, Garcia-Molina H: Correcting for missing data in information cascades. Proc WSDM'11 2011, 55-64.

44. Riley S: Large-scale spatial-transmission models of infectious disease. Science 2007, :1298-1301. doi:10.1126/science.1134695.

45. Colizza V, Pastor-Satorras R, Vespignani A: Reaction-diffusion processes and metapopulation models in heterogeneous networks. Nature Phys 2007, 3:276-282.

46. Grenfell BT, Bjornstad ON, Kappey J: Travelling waves and spatial hierarchies in measles epidemics. Nature 2001, 414:716-723.

47. Eubank S, Guclu H, Kumar VSA, Marathe MV, Srinivasan A, Toroczkai Z, Wang $\mathrm{N}$ : Modelling disease outbreaks in realistic urban social networks. Nature 2004, 429:180-184.

48. Eames KTD, Keeling MJ: Contact tracing and disease control. Proc Roy Soc B 2003, 270:2565-2571.

49. Read JM, Keeling MJ: Disease evolution on networks: the role of contact structure. Proc Roy Soc B 2003, 270:699-708.

50. Riolo CS, Koopman JS, Chick SE: Methods and measures for the description of epidemiologic contact networks. J Urban Health 2001, 78(3):446-457.

51. Liljeros F, Edling CR, Amaral LAN, Stanley HE, Aberg Y: The web of human sexual contacts. Nature 2001, 411:907-908.
52. Zelner JL, King $A A$, Moe $C L$, Eisenberg JNS: How infections propagate after point-source outbreak - An analysis of secondary norovirus transmission. Epidem 2010, 21(5):711-717.

53. Hohle $M$, Jorgenson $E, O^{\prime}$ Neill PD: Inference in disease transmission experiments by using stochastic epidemic models. J App/ Stat 2005, 54:349-66.

54. Meyers S, Leskovec J: On the convexity of latent social network inference. Adv Neur In 2010, 1741:1749.

55. Eagle N, Pentland AS, Lazer D: Inferring friendship network structure by using mobile phone data. Proc Nat Acad Sci 2009, 106(36):15274-15278.

56. Choudhury M, Mason WA, Hofman JM, Watts DJ: Inferring relevant social networks from interpersonal communication. Proc WWW'10 2010, 301-310

57. Liben-Nowell D, Kleinberg J: The link-prediction problem for social networks. J Am Soc Info Sci Tech 2007, 58:1019-1031.

58. Getoor L, Friedman N, Koller D, Taskar B: Learning probabilistic models of link structure. J Machine Learn Res 2002, 3:690-707.

59. Giot L, Bader J, Brouwer C, Chaudhuri A, Kuang B, Li Y, Hao Y, Ooi C, Godwin $B$, et al: A protein interaction map of Drosophila melanogaster. Science 2003, 302(5651):1727.

60. Middendorf M, Ziv E, Wiggins C: Inferring network mechanisms: the Drosophila melanogaster protein interaction network. Proc Nat Acad Sci 2005, 102(9):3192.

61. Nemhauser G, Wolsey L, Fisher M: An analysis of the approximations for maximizing submodular set functions. Math Progr 1978, 14(1):265-294.

62. Yang G, Gao Q, Zhou S, Malone JB, McCarroll JC, Tanner M, Vounatsou P, Bergquist $\mathrm{R}$, Utzinger J, Zhou X: Mapping and predicting malaria transmission in the People's Republic of China using integrated biologydriven and statistical models. Geosp Health 2010, 5(1):11-22.

63. Clements ACA, Barnett AG, Cheng ZW: Space-time variation of malaria incidence in Yunnan province. China Malar J 2009, 8:180.

64. Xiao D, Long Y, Wang S, Fang L, Xu D, Wang G, Li L, Cao W, Yan Y: Spatiotemporal distribution of malaria and the association between its epidemic and climate factors in Hainan. China Malar J 2010, 9:185.

65. Liu J, Jin X, Tsui KC: Autonomy Oriented Computing: From Problem Solving to Complex Systems Modelling. Berlin: Springer; 2004

66. Liu J: Autonomy-oriented computing (AOC): The nature and implications of a paradigm for self-organized computing. Proc. ICNC'08. New York: IEEE Press; 2008:3-11

67. Liu J, Jin X, Tsui KC: Autonomy oriented computing (AOC): Formulating computational systems with autonomous components. IEEE Trans Sys Man Cyb A 2005, 35(6):879-902.

68. Bailey NTJ: The Mathematical Theory of Infectious Diseases and its Applications. New York: Hafner Press; 1975.

69. Anderson RM, May RM: Infectious Diseases in Human. Oxford: Oxford University Press; 1992.

70. Diekmann O, Heesterbeek JAP: Mathematical Epidemiology of Infectious Diseases: Model Building Analysis and Interpretation. New York: John Wiley \& Son Publisher; 2000.

71. Granovetter M: Threshold models of collective behaviour. Am J Socio 1978, 83(6):1420-1443.

72. Valente T: Network Models of the Diffusion of Innovations. New York: Hampton Press; 1995.

73. Erdos P, Renyi A: On the evolution of random graphs. Publ Math Inst Hungarian Acad Sci 1960, 5:17-60

74. Gomez-Rodriguez M, Balduzzi D, Scholkopf B: Uncovering the temporal dynamics of diffusion networks. Proc ICML'11 2011, 561-568.

75. Mandal S, Sarkar RR, Sinha S: Mathematical models of malaria - a review. Malar J 2011, 10:202

doi:10.1186/2049-9957-1-11

Cite this article as: Liu et al: Malaria transmission modelling: a network perspective. Infectious Diseases of poverty 2012 1:11. 